\title{
The Zakharov-Kuznetsov equation in high dimensions: small initial data of critical regularity
}

\author{
Sebastian Herr And Shinya KinOShita
}

Abstract. The Zakharov-Kuznetsov equation in spatial dimension $d \geq 5$ is considered. The Cauchy problem is shown to be globally well-posed for small initial data in critical spaces, and it is proved that solutions scatter to free solutions as $t \rightarrow \pm \infty$. The proof is based on i) novel endpoint non-isotropic Strichartz estimates which are derived from the $(d-1)$-dimensional Schrödinger equation, ii) transversal bilinear restriction estimates, and iii) an interpolation argument in critical function spaces. Under an additional radiality assumption, a similar result is obtained in dimension $d=4$.

\section{Introduction}

This paper is concerned with the Zakharov-Kuznetsov equation

$$
\begin{array}{ll}
\partial_{t} u+\partial_{x_{1}} \Delta u=\partial_{x_{1}} u^{2} & \text { in } \mathbb{R} \times \mathbb{R}^{d} \\
u(0, \cdot)=u_{0} & \text { on } \mathbb{R}^{d}
\end{array}
$$

where $d \geq 2, u=u(t, x),(t, x)=\left(t, x_{1}, \ldots, x_{d}\right) \in \mathbb{R} \times \mathbb{R}^{d}, u$ is real-valued, and $\Delta$ denotes the Laplacian with respect to $x$.

The Zakharov-Kuznetsov equation was introduced in [13] as a model for propagation of ion-sound waves in magnetic fields. The Zakharov-Kuznetsov equation can be seen as a multidimensional extension of the well-known Korteweg-de Vries (KdV) equation. In contrast to the $\mathrm{KdV}$ equation, the Zakharov-Kuznetsov equation is not completely integrable, but possesses two invariants,

$$
M(u):=\int_{\mathbb{R}^{d}} u^{2} d x, \quad E(u):=\int_{\mathbb{R}^{d}} \frac{1}{2}|\nabla u|^{2}+\frac{1}{3} u^{3} d x .
$$

In the following, $H^{s}\left(\mathbb{R}^{d}\right)$ denotes the standard $L^{2}$-based inhomogeneous Sobolev space and $B_{2,1}^{s}\left(\mathbb{R}^{d}\right)$ is the Besov refinement, and the dotted versions their homogeneous

Mathematics Subject Classification: 35Q53, 35A01

Keywords: Global well-posedness, scattering, Zakharov-Kuznetsov equation.

Financial support by the German Research Foundation (DFG) through the CRC 1283 "Taming uncertainty and profiting from randomness and low regularity in analysis, stochastics and their applications" is acknowledged. 
counterparts, see below for definitions. The scale-invariant regularity threshold for (1.1) is $s_{c}=\frac{d-4}{2}$.

Before we state our main results, let us briefly summarize the progress which has been made regarding the well-posedness problem associated to (1.1). In the twodimensional case, Faminskiı̌ [3] established global well-posedness in the energy space $H^{1}\left(\mathbb{R}^{2}\right)$. Later, Linares and Pastor [9] proved local well-posedness in $H^{s}\left(\mathbb{R}^{2}\right)$ for $s>3 / 4$; before Grünrock and Herr [4] and Molinet and Pilod [11] showed local wellposedness for $s>1 / 2$. Recently, the second author [7] proved local well-posedness for $s>-1 / 4$. In dimension $d=3$, Linares and Saut [10] obtained local well-posedness in $H^{s}\left(\mathbb{R}^{3}\right)$ for $s>9 / 8$. Ribaud and Vento [12] proved local well-posedness for $s>1$ and in $B_{2}^{1,1}\left(\mathbb{R}^{3}\right)$. The global well-posedness in $H^{s}\left(\mathbb{R}^{3}\right)$ for $s>1$ was obtained by Molinet and Pilod in [11]. Recently, in dimensions $d \geq 3$, local well-posedness in $H^{s}\left(\mathbb{R}^{d}\right)$ in the full subcritical range $s>s_{c}$ was proved in [5], which implies global well-posedness in $H^{1}\left(\mathbb{R}^{d}\right)$ if $3 \leq d \leq 5$ and in $L^{2}\left(\mathbb{R}^{3}\right)$. We refer the reader to these papers for a more thorough account on the Zakharov-Kuznetsov equation and more references.

In the present paper, we address the problem of global well-posedness and scattering for small initial data in critical spaces. By well-posedness we mean existence of a (mild) solution, uniqueness of solutions (in some subspace) and (locally Lipschitz) continuous dependence of solutions on the initial data. We say that a global solution $u \in C\left(\mathbb{R}, H^{s}\left(\mathbb{R}^{d}\right)\right)$ of (1.1) scatters as $t \rightarrow \pm \infty$, if there exist $u_{ \pm} \in H^{s}\left(\mathbb{R}^{d}\right)$ such that

$$
\left\|u(t)-e^{t S} u_{ \pm}\right\|_{H^{s}\left(\mathbb{R}^{d}\right)} \rightarrow 0 \quad(t \rightarrow \pm \infty) .
$$

Here, $e^{t S}$ denotes the unitary group generated by the skew-adjoint linear operator $S=-\partial_{x_{1}} \Delta$, so that $e^{t S} u_{ \pm}$solves the linear homogeneous equation.

Our first main result covers small data in dimension $d=5$.

Theorem 1.1. For $d=5$, the Cauchy problem (1.1) is globally well-posed for small initial data in $B_{2,1}^{S_{c}}\left(\mathbb{R}^{5}\right)$, and solutions scatter as $t \rightarrow \pm \infty$. The same result holds in $\dot{B}_{2,1}^{s_{c}}\left(\mathbb{R}^{d}\right)$.

In dimensions $d \geq 6$, we can extend this to Sobolev regularity.

Theorem 1.2. For $d \geq 6$, the Cauchy problem (1.1) is globally well-posed for small initial data in $H^{s_{c}}\left(\mathbb{R}^{d}\right)$, and solutions scatter as $t \rightarrow \pm \infty$. The same result holds in $\dot{H}^{s_{c}}\left(\mathbb{R}^{d}\right)$.

Note that in $d=6$ this result includes the energy space $\dot{H}^{1}\left(\mathbb{R}^{6}\right)$.

If we restrict to initial data which is radial in the last $(d-1)$ variables (see below for definitions), we obtain small data global well-posedness and scattering in the critical Sobolev spaces for any dimension $d \geq 4$.

Theorem 1.3. For $d \geq 4$, the Cauchy problem (1.1) is globally well-posed for small data in $H_{\mathrm{rad}}^{s_{c}}\left(\mathbb{R}^{d}\right)$, and solutions scatter as $t \rightarrow \pm \infty$. The same result holds for radial data in $\dot{H}_{\mathrm{rad}}^{s_{c}}\left(\mathbb{R}^{d}\right)$. 
As the proof shows, the radiality assumption can be weakened to an angular regularity assumption, but we do not pursue this. One of the most interesting special cases here is $d=4$, when $s_{c}=0$; hence, the result covers the radial $L^{2}$ space.

The main idea of this paper is to combine a new set of non-isotropic Strichartz estimates with the bilinear transversal estimate and an interpolation argument in critical function spaces.

The paper is structured as follows: In Sect. 1.1 we introduce notation. In Sect. 2 we derive Strichartz-type estimates which are based on the well-known Strichartz estimates for the $(d-1)$-dimensional Schrödinger equation and allow us to treat the case $d=5$. In Sect. 3 we combine this with the bilinear transversal estimate and an interpolation argument, which leads to a proof of Theorem 1.2. Finally, in Sect. 4 we discuss an variation of these ideas under the additional radiality assumption and a proof of Theorem 1.3.

\subsection{Notation}

We write $x^{\prime}=\left(x_{2}, \ldots, x_{d}\right), D_{x_{j}}=-i \partial_{j}, D=(-\Delta)^{\frac{1}{2}},\left|\nabla_{x^{\prime}}\right|^{s}=\mathcal{F}_{x^{\prime}}^{-1}\left|\xi^{\prime}\right|^{s} \mathcal{F}_{x^{\prime}}$, and $\left\langle\nabla_{x^{\prime}}\right\rangle^{s}=\mathcal{F}_{x^{\prime}}^{-1}\left\langle\xi^{\prime}\right\rangle^{s} \mathcal{F}_{x^{\prime}}$. Here and in the sequel, we denote the Fourier transform of $u$ in time, space, and the first spatial variable, by $\mathcal{F}_{t} u, \mathcal{F}_{x} u, \mathcal{F}_{x_{1}} u$, and $\mathcal{F}_{x^{\prime}}$, respectively. $\mathcal{F}_{t, x} u=\widehat{u}$ denotes the Fourier transform of $u$ in time and space. Choose a nonnegative bump function $\psi \in C_{c}^{\infty}(\mathbb{R})$ supported in the interval $(1 / 2,2)$ with the property that $\sum_{N \in 2^{\mathbb{Z}}} \psi(r / N)=1$ for $r>0$, and set $\psi_{N}=\psi(\cdot / N)$. For $N, \lambda, M \in 2^{\mathbb{Z}}$, we define (spatial) frequency projections $P_{N}, Q_{\lambda}$, and $R_{M}$ as the Fourier multipliers with symbols $\psi_{N}(|\xi|), \psi_{\lambda}\left(\left|\xi_{1}\right|\right)$, and $\psi_{M}\left(\left|\xi^{\prime}\right|\right)$, respectively, where $(\tau, \xi)=\left(\tau, \xi_{1}, \xi^{\prime}\right)=$ $\left(\tau, \xi_{1}, \ldots, \xi_{d}\right) \in \mathbb{R} \times \mathbb{R}^{d}$ are temporal and spatial frequencies. In addition, we define

$$
P_{\leq 1}=\sum_{1 \geq N \in 2^{\mathbb{Z}}} P_{N}, \quad Q_{\leq 1}:=\sum_{1 \geq \lambda \in 2^{\mathbb{Z}}} Q_{\lambda}, \text { and } R_{\leq 1}:=\sum_{1 \geq M \in 2^{\mathbb{Z}}} R_{M} .
$$

As usual, the Sobolev space $H^{s}\left(\mathbb{R}^{d}\right)$ is defined as the completion of the Schwartz functions with respect to the norm

$$
\|f\|_{H^{s}\left(\mathbb{R}^{d}\right)}=\left(\int_{\mathbb{R}^{d}}\langle\xi\rangle^{2 s}|\widehat{f}(\xi)|^{2} d \xi\right)^{\frac{1}{2}}
$$

and the (smaller) Besov space $B_{2,1}^{s}\left(\mathbb{R}^{d}\right)$ as the completion of the Schwartz functions $\mathcal{S}\left(\mathbb{R}^{d}\right)$ with respect to the norm

$$
\|f\|_{B_{2,1}^{s}\left(\mathbb{R}^{d}\right)}=\left\|P_{\leq 1} f\right\|_{L^{2}}+\sum_{N \in 2^{\mathbb{N}}} N^{s}\left\|P_{N} f\right\|_{L^{2}} .
$$

Similarly, for $s \geq 0$, the homogeneous Sobolev space $\dot{H}^{s}\left(\mathbb{R}^{d}\right)$ is defined as the completion of the Schwartz functions with respect to the norm

$$
\|f\|_{\dot{H}^{s}\left(\mathbb{R}^{d}\right)}=\left(\int_{\mathbb{R}^{d}}|\xi|^{2 s}|\widehat{f}(\xi)|^{2} d \xi\right)^{\frac{1}{2}},
$$


and the homogeneous Besov space $\dot{B}_{2,1}^{s}\left(\mathbb{R}^{d}\right)$ as the completion of the Schwartz functions with respect to the norm

$$
\|f\|_{\dot{B}_{2,1}^{s}\left(\mathbb{R}^{d}\right)}=\sum_{N \in 2^{\mathbb{Z}}} N^{s}\left\|P_{N} f\right\|_{L^{2}}
$$

The radial subspaces $H_{\text {rad }}^{s}\left(\mathbb{R}^{d}\right)$ and $\dot{H}_{\text {rad }}^{s}\left(\mathbb{R}^{d}\right)$ are defined by the requirement that $f\left(x_{1}, x^{\prime}\right)=f\left(x_{1}, y^{\prime}\right)$ if $\left|x^{\prime}\right|=\left|y^{\prime}\right|$, i.e., for fixed $x_{1}$, the functions are radial in $x^{\prime}$.

Finally, the Duhamel operator is denoted by

$$
\mathcal{I}(F)(t):=\int_{0}^{t} e^{\left(t-t^{\prime}\right) S} F\left(t^{\prime}\right) d t^{\prime}
$$

\section{Strichartz estimates and the proof of Theorem 1.1}

For $d \geq 2$, we say $(q, r)$ is $(d-1)$-admissible if

$$
2 \leq q, r \leq \infty, 2 / q=(d-1)(1 / 2-1 / r),(d, q, r) \neq(3,2, \infty)
$$

Theorem 2.1. Let $d \geq 2$ and $\left(q_{1}, r_{1}\right),\left(q_{2}, r_{2}\right)$ be $(d-1)$-admissible. Then, we have

$$
\begin{aligned}
& \left\|D_{x_{1}}^{\frac{1}{q}} e^{t S} f\right\|_{L_{t}^{q_{1}} L_{x^{\prime}}^{r_{1}} L_{x_{1}}^{2}} \lesssim\|f\|_{L_{x}^{2} .} \\
& \left\|D_{x_{1}}^{\frac{1}{q_{1}}+\frac{1}{q_{2}}} \mathcal{I} F\right\|_{L_{t}^{q_{1}} L_{x^{\prime}}^{r_{1}} L_{x_{1}}^{2}} \lesssim\|F\|_{L_{t}^{q_{2}^{\prime}} L_{x^{\prime}}^{r_{2}^{\prime}} L_{x_{1}}^{2}},
\end{aligned}
$$

where $1 / q_{2}^{\prime}=1-1 / q_{2}$ and $1 / r_{2}^{\prime}=1-1 / r_{2}$.

Proof. Let $\Delta_{x^{\prime}}=\sum_{j=2}^{d} \partial_{x_{j}}^{2}$. For fixed $\xi_{1} \in \mathbb{R}$, define $V_{\xi_{1}}(t) f\left(x^{\prime}\right):=\left(e^{-i t \xi_{1} \Delta_{x^{\prime}}} f\right)\left(x^{\prime}\right)$. Since $V_{\xi_{1}}\left(t / \xi_{1}\right)=e^{i t \Delta_{x^{\prime}}}$, for $f \in \mathcal{S}\left(\mathbb{R}^{d-1}\right)$ and $F \in \mathcal{S}\left(\mathbb{R} \times \mathbb{R}^{d-1}\right)$, the Strichartz estimates of Schrödinger equations in $\mathbb{R}^{d-1}$ imply

$$
\begin{aligned}
&\left\|\left|\xi_{1}\right|^{\frac{1}{q_{1}}} V_{\xi_{1}}(t) f\right\|_{L_{t}^{q_{1}} L_{x^{\prime}}^{r_{1}}} \lesssim\|f\|_{L_{x^{\prime}}^{2}}, \\
&\left\|\int_{0}^{t}\left|\xi_{1}\right|^{\frac{1}{q_{1}}+\frac{1}{q_{2}}} V_{\xi_{1}}\left(t-t^{\prime}\right) F\left(t^{\prime}\right) d t^{\prime}\right\|_{L_{t}^{q_{1}} L_{x^{\prime}}^{r_{1}}} \lesssim\|F\|_{L_{t}^{q_{2}^{\prime}} L_{x^{\prime}}^{r_{2}^{\prime}},}
\end{aligned}
$$

see [6, Theorem 1.2] for details. We deduce from Plancherel's theorem, Minkowski's inequality and (2.3) that

$$
\left\|D_{x_{1}}^{\frac{1}{q}} e^{t S} f\right\|_{L_{t}^{q} L_{x^{\prime}}^{r} L_{x_{1}}^{2}}=\left(\int_{\mathbb{R}}\left\|\left|\xi_{1}\right|^{\frac{1}{q}} V_{\xi_{1}} \mathcal{F}_{x_{1}} f\right\|_{L_{t}^{q} L_{x^{\prime}}^{r}}^{2} d \xi_{1}\right)^{\frac{1}{2}} \lesssim\|f\|_{L_{x}^{2}},
$$


which is (2.1). Similarly, by (2.4),

$$
\begin{aligned}
& \left\|D_{x_{1}}^{\frac{1}{q_{1}}+\frac{1}{q_{2}}} \mathcal{I}(F)\right\|_{L_{t}^{q_{1}} L_{x^{\prime}}^{r_{1}} L_{x_{1}}^{2}} \\
& \quad=\left(\int_{\mathbb{R}}\left\|\int_{0}^{t} \mid \xi_{1} \frac{1}{q_{1}}+\frac{1}{q_{2}} V_{\xi_{1}}\left(t-t^{\prime}\right) \mathcal{F}_{x_{1}}(F)\left(t^{\prime}\right) d t^{\prime}\right\|_{L_{t}^{q_{1}} L_{x^{\prime}}^{r_{1}}}^{2} d \xi_{1}\right)^{\frac{1}{2}} \\
& \quad \lesssim\|F\|_{L_{t}^{q_{2}^{\prime}} L_{x^{\prime}}^{r_{2}^{\prime}} L_{x_{1}}^{2}},
\end{aligned}
$$

which is (2.2).

Now, we can complete the proof of Theorem 1.1. Recall that $d=5$ implies $s_{C}=1 / 2$.

Definition 2.2. We define

$$
\begin{aligned}
\|u\|_{Z^{\frac{1}{2}}}:= & \left\|P_{\leq 1} u\right\|_{L_{t}^{\infty} L_{x}^{2}}+\left\|P_{\leq 1} D_{x_{1}}^{\frac{1}{2}} u\right\|_{L_{t}^{2} L_{x^{\prime}}^{4} L_{x_{1}}^{2}} \\
& +\sum_{N \in 2^{\mathbb{N}}} N^{\frac{1}{2}}\left(\left\|P_{N} u\right\|_{L_{t}^{\infty} L_{x}^{2}}+\left\|P_{N} D_{x_{1}}^{\frac{1}{2}} u\right\|_{L_{t}^{2} L_{x^{\prime}}^{4} L_{x_{1}}^{2}}\right), \\
\|u\|_{\dot{Z}^{\frac{1}{2}}}:= & \sum_{N \in 2^{\mathbb{Z}}} N^{\frac{1}{2}}\left(\left\|P_{N} u\right\|_{L_{t}^{\infty} L_{x}^{2}}+\left\|P_{N} D_{x_{1}}^{\frac{1}{2}} u\right\|_{L_{t}^{2} L_{x^{\prime}}^{4} L_{x_{1}}^{2}}\right),
\end{aligned}
$$

and the corresponding Banach spaces.

By the standard argument involving the contraction mapping principle, it suffices to prove the following:

Proposition 2.3. Let $d=5$. Then, we have

$$
\left\|\mathcal{I}\left(\partial_{x_{1}}\left(u_{1} u_{2}\right)\right)\right\|_{Z^{\frac{1}{2}}} \lesssim\left\|u_{1}\right\|_{Z^{\frac{1}{2}}}\left\|u_{2}\right\|_{Z^{\frac{1}{2}}}, \quad\left\|\mathcal{I}\left(\partial_{x_{1}}\left(u_{1} u_{2}\right)\right)\right\|_{\dot{Z}^{\frac{1}{2}}} \lesssim\left\|u_{1}\right\|_{\dot{Z}^{\frac{1}{2}}}\left\|u_{2}\right\|_{\dot{Z}^{\frac{1}{2}}} \cdot
$$

Proof. Let $N_{\max }=\max \left(N_{1}, N_{2}, N_{3}\right)$ and $N_{\min }=\min \left(N_{1}, N_{2}, N_{3}\right)$. Theorem 2.1 gives

$$
\begin{aligned}
& \left.\left\|P_{N_{3}} \mathcal{I}\left(\partial_{x_{1}}\left(u_{1} u_{2}\right)\right)\right\|\right|_{L_{t}^{\infty} L_{x}^{2}}+\left\|P_{N_{3}} D_{x_{1}}^{\frac{1}{2}} \mathcal{I}\left(\partial_{x_{1}}\left(u_{1} u_{2}\right)\right)\right\|_{L_{t}^{2} L_{x^{\prime}}^{4} L_{x_{1}}^{2}} \\
& \lesssim\left\|P_{N_{3}} D_{x_{1}}^{\frac{1}{2}}\left(u_{1} u_{2}\right)\right\|_{L_{t}^{2} L_{x^{\prime}}^{\frac{4}{3}} L_{x_{1}}^{2}} \cdot
\end{aligned}
$$

We observe that $P_{N_{3}}=P_{N_{3}} Q_{\leq N_{3}}$. Since the kernel of the operator $P_{N_{3}}$ is uniformly bounded in $L_{x}^{1}$, we obtain

$$
\left\|P_{N_{3}} D_{x_{1}}^{\frac{1}{2}}\left(u_{1} u_{2}\right)\right\|_{L_{t}^{2} L_{x^{\prime}}^{2} L_{x_{1}}^{2}} \lesssim\left\|Q_{\leq N_{3}} D_{x_{1}}^{\frac{1}{2}}\left(u_{1} u_{2}\right)\right\|_{L_{t}^{2} L_{x^{\prime}}^{2} L_{x_{1}}^{2}}^{\frac{4}{2}} .
$$

Now, by freezing $\left(t, x^{\prime}\right)$, we can use Plancherel's theorem and Bernstein's inequality in $x_{1}$ to compute

$$
\left\|Q_{\leq N_{3}} D_{x_{1}}^{\frac{1}{2}}\left(u_{N_{1}} u_{N_{2}}\right)\right\|_{L_{x_{1}}^{2}} \lesssim N_{\min }^{\frac{1}{2}}\left(\left\|D_{x_{1}}^{\frac{1}{2}} u_{N_{1}}\right\|_{L_{x_{1}}^{2}}\left\|u_{N_{2}}\right\|_{L_{x_{1}}^{2}}+\left\|u_{N_{1}}\right\|_{L_{x_{1}}^{2}}\left\|D_{x_{1}}^{\frac{1}{2}} u_{N_{2}}\right\|_{L_{x_{1}}^{2}}\right)
$$


Finally, Hölder's inequality with respect to $\left(t, x^{\prime}\right)$ implies

$$
\begin{aligned}
& \left\|P_{N_{3}} D_{x_{1}}^{\frac{1}{2}}\left(u_{N_{1}} u_{N_{2}}\right)\right\|_{L_{t}^{2} L_{x^{\prime}}^{\frac{4}{3}} L_{x_{1}}^{2}} \\
& \quad \lesssim N_{\min }^{\frac{1}{2}}\left\|D_{x_{1}}^{\frac{1}{2}} u_{N_{1}}\right\|_{L_{t}^{2} L_{x^{\prime}}^{4} L_{x_{1}}^{2}}\left\|u_{N_{2}}\right\|_{L_{t}^{\infty} L_{x}^{2}}+N_{\min }^{\frac{1}{2}}\left\|u_{N_{1}}\right\|_{L_{t}^{\infty} L_{x}^{2}}\left\|D_{x_{1}}^{\frac{1}{2}} u_{N_{2}}\right\|_{L_{t}^{2} L_{x^{\prime}}^{4} L_{x_{1}}^{2}} .
\end{aligned}
$$

This can be summed up both in the homogeneous and in the inhomogeneous version.

This argument also implies the scattering claim, since it implies that the Duhamel integral converges to a free solutions as $t \rightarrow \pm \infty$. We omit the details of this standard argument.

\section{Transversal estimates and the proof of Theorem 1.2}

Lemma 3.1. Let $d \geq 2$ and $f_{N_{1}, \lambda_{1}}=Q_{\lambda_{1}} P_{N_{1}} f, g_{N_{2}, \lambda_{2}}=Q_{\lambda_{2}} P_{N_{2}}$ g. Forall $\lambda_{j}, N_{j} \in$ $2^{\mathbb{Z}}$ such that

$$
|\nabla \varphi(\xi)-\nabla \varphi(\eta)| \gtrsim \max \left\{\lambda_{1}, \lambda_{2}\right\} N_{\max }
$$

for all $\xi \in \operatorname{supp} \widehat{f}_{N_{1}, \lambda_{1}}, \eta \in \operatorname{supp} \widehat{g}_{N_{2}, \lambda_{2}}$, it holds that

$$
\left\|P_{N_{3}}\left(e^{t S} f_{N_{1}, \lambda_{1}} e^{t S} g_{N_{2}, \lambda_{2}}\right)\right\|_{L_{t}^{2} L_{x}^{2}} \lesssim\left(\frac{N_{\min }^{d-1}}{\max \left\{\lambda_{1}, \lambda_{2}\right\} N_{\max }}\right)^{\frac{1}{2}}\left\|f_{N_{1}, \lambda_{1}}\right\|_{L^{2}}\left\|g_{N_{2}, \lambda_{2}}\right\|_{L^{2}} .
$$

This is an instance of the well-known bilinear transversal estimate, e.g., a special case of [1, Lemma 2.6], where a proof can be found.

Next, we recall the definitions of $U^{p}$ and $V^{p}$ spaces, which have been introduced in [8] the dispersive PDE context. We refer the reader to [1] and the references therein for further details. For $1 \leq p<\infty$, we call a function $a: \mathbb{R} \rightarrow L^{2}\left(\mathbb{R}^{d}\right)$ a $p$-atom, if there exists a finite partition $\mathcal{J}=\left\{\left(-\infty, t_{1}\right),\left[t_{2}, t_{3}\right), \ldots,\left[t_{K}, \infty\right)\right\}$ of the real line such that

$$
a(t)=\sum_{J \in \mathcal{J}} \mathbf{1}(t) f_{J}, \quad \sum_{J \in \mathcal{J}}\left\|f_{J}\right\|_{L^{2}}^{p} \leq 1
$$

Now, $U^{p}$ is defined as the space of all $u: \mathbb{R} \rightarrow L^{2}\left(\mathbb{R}^{d}\right)$, such that there exists an atomic decomposition $u=\sum_{j=1}^{\infty} c_{j} a_{j}$, where $\left(c_{j}\right) \in \ell^{1}(\mathbb{N})$ and the $a_{j}$ 's are $p$-atoms. Then, $\|u\|_{U^{p}}=\inf \sum_{j=1}^{\infty}\left|c_{j}\right|$ is a norm (the infimum is taken with respect to all possible atomic decompositions), so that $U^{p}$ is a Banach space. Further, let $V^{p}$ denote the space of all right-continuous functions $v: \mathbb{R} \rightarrow L^{2}\left(\mathbb{R}^{d}\right)$, such that

$$
\|v\|_{V^{p}}=\|v\|_{L_{t}^{\infty} L_{x}^{2}}+\sup \left(\sum_{j \in \mathbb{Z}}\left\|v\left(t_{j}\right)-v\left(t_{j-1}\right)\right\|_{L_{x}^{2}}^{p}\right)^{\frac{1}{p}}<\infty,
$$


where the supremum is taken over all increasing sequences $\left(t_{j}\right)$. Now, we define the atomic space $U_{S}^{p}=e^{\cdot S} U^{p}$ with norm $\|u\|_{U_{S}^{p}}=\left\|e^{-\cdot S} u\right\|_{U^{p}}$, and $V_{S}^{p}=e^{\cdot S} V^{p}$ with norm $\|u\|_{V_{S}^{p}}=\left\|e^{-\cdot S} u\right\|_{V^{p}}$.

There is the embedding $V_{S}^{p} \subset U_{S}^{q}$ if $p<q$, see [8, Lemma 6.4]. Due to the atomic structure of $U_{S}^{q}$ and the Strichartz estimate (2.1), we have

$$
\left\|D_{x_{1}}^{\frac{1}{q}} u\right\|_{L_{t}^{q} L_{x^{\prime}}^{r} L_{x_{1}}^{2}} \lesssim\|u\|_{U_{S}^{q}}
$$

for $(d-1)$-admissible pairs, and $\|u\|_{U_{S}^{q}}$ may be replaced by $\|u\|_{V_{S}^{2}}$ for non-endpoint pairs, i.e., when $q>2$.

Let $\lambda_{\max }:=\max \left(\lambda_{1}, \lambda_{2}, \lambda_{3}\right)$ and $\lambda_{\min }:=\min \left(\lambda_{1}, \lambda_{2}, \lambda_{3}\right)$. We use the shorthand notation $u_{N}:=P_{N} u, u_{N, \lambda}:=Q_{\lambda} P_{N} u$, etc.

Proposition 3.2. Let $d \geq 6$ and the pair $(q, r)$ be $(d-1)$-admissible with $2<q<$ $\frac{2(d-3)}{d-5}$, and let $\varepsilon>0$. Suppose

$$
|\nabla \varphi(\xi)-\nabla \varphi(\eta)| \gtrsim \max \left\{\lambda_{1}, \lambda_{2}\right\} N_{\max }
$$

for all $\xi \in \operatorname{supp}_{\xi} \widehat{u}_{N_{1}, \lambda_{1}}, \eta \in \operatorname{supp}_{\xi} \widehat{v}_{N_{2}, \lambda_{2}}$. Then, for all $\lambda_{j}, N_{j} \in 2^{\mathbb{Z}}$,

$$
\begin{aligned}
& \left\|P_{N_{3}} Q_{\lambda_{3}}\left(u_{N_{1}, \lambda_{1}} v_{N_{2}, \lambda_{2}}\right)\right\|_{L_{t}^{q^{\prime}} L_{x^{\prime}}^{r^{\prime}} L_{x_{1}}^{2}} \\
& \lesssim \lambda_{\max }^{-\frac{1}{2}+\frac{2 \varepsilon}{d-3}} \lambda_{\min }^{-\frac{1}{2}+\frac{1}{d-3}+\frac{1}{q}-\frac{2 \varepsilon}{d-3}} N_{\max }^{-\frac{1}{2}+\frac{1}{d-3}} N_{\min }^{\frac{d-3}{2}-\frac{2}{d-3}}\left\|u_{N_{1}, \lambda_{1}}\right\|_{V_{S}^{2}}\left\|v_{N_{2}, \lambda_{2}}\right\|_{V_{S}^{2}} .
\end{aligned}
$$

Proof. By symmetry, we may assume that $\lambda_{1} \sim \lambda_{\max }$. For a sufficiently small $\varepsilon>0$, we define the $(d-1)$-admissible pairs $\left(q_{1}, r_{1}\right)$ and $\left(q_{2}, r_{2}\right)$ by

$$
\begin{aligned}
\left(\frac{1}{q_{1}}, \frac{1}{r_{1}}\right) & =\left(\frac{1}{2}-\varepsilon, \frac{1}{2}-\frac{1-2 \varepsilon}{d-1}\right),\left(\frac{1}{q_{2}}, \frac{1}{r_{2}}\right) \\
& =\left(\frac{d-3}{4}-\frac{d-3}{2 q}+\varepsilon, \frac{d-3}{q(d-1)}+\frac{1-2 \varepsilon}{d-1}\right) .
\end{aligned}
$$

In addition, letting

$$
\frac{1}{\alpha}=\frac{1}{q_{1}}+\frac{1}{q_{2}}, \quad \frac{1}{\beta}=\frac{1}{r_{1}}+\frac{1}{r_{2}},
$$

by using (3.2), we have

$$
\begin{aligned}
& \left\|P_{N_{3}} Q_{\lambda_{3}}\left(u_{N_{1}, \lambda_{1}} v_{N_{2}, \lambda_{2}}\right)\right\|_{L_{t}^{\alpha} L_{x^{\prime}}^{\beta} L_{x_{1}}^{2}} \lesssim \lambda_{\min }^{\frac{1}{2}}\left\|u_{N_{1}, \lambda_{1}}\right\|_{L_{t}^{q_{1}} L_{x^{\prime}}^{r_{1}} L_{x_{1}}^{2}}\left\|v_{N_{2}, \lambda_{2}}\right\|_{L_{t}^{q_{2}} L_{x^{\prime}}^{r_{2}} L_{x_{1}}^{2}} \\
& \lesssim \lambda_{\min }^{\frac{1}{2}-\frac{1}{q_{2}}} \lambda_{\max }^{-\frac{1}{q_{1}}}\left\|u_{N_{1}, \lambda_{1}}\right\|_{U_{S}^{q_{1}}}\left\|v_{N_{2}, \lambda_{2}}\right\|_{U_{S}^{q_{2}}}
\end{aligned}
$$

Lemma 3.1 immediately extends from free solutions to $2-$ atoms. Therefore, the atomic structure of $U^{2}$ implies

$$
\left\|P_{N_{3}} Q_{\lambda_{3}}\left(u_{N_{1}, \lambda_{1}} v_{N_{2}, \lambda_{2}}\right)\right\|_{L_{t}^{2} L_{x}^{2}} \lesssim\left(\frac{N_{\min }^{d-1}}{\lambda_{\max } N_{\max }}\right)^{\frac{1}{2}}\left\|u_{N_{1}, \lambda_{1}}\right\|_{U_{S}^{2}}\left\|v_{N_{2}, \lambda_{2}}\right\|_{U_{S}^{2}}
$$




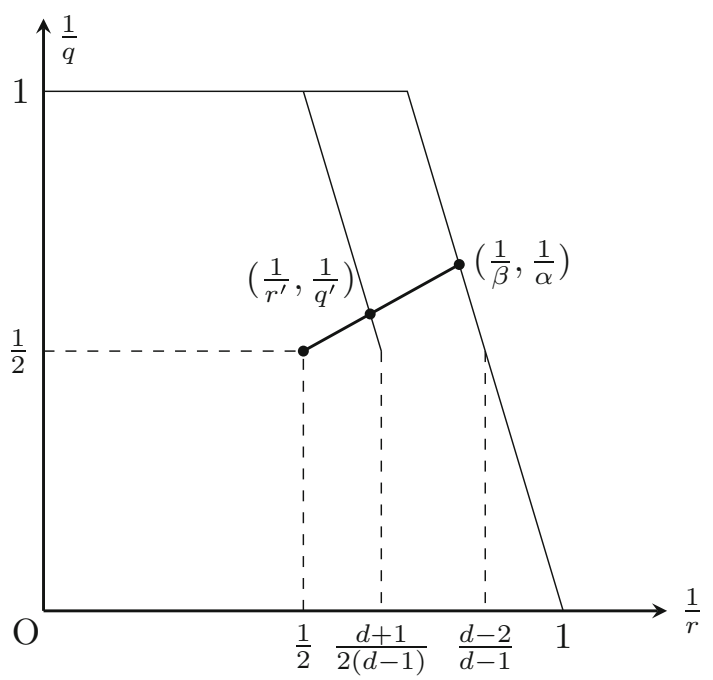

Figure 1. Choice of parameters in the interpolation argument

For $\theta=\frac{2}{d-3}$, it is observed that

$$
\frac{\theta}{\alpha}+\frac{1-\theta}{2}=1-\frac{1}{q}\left(=: \frac{1}{q^{\prime}}\right), \quad \frac{\theta}{\beta}+\frac{1-\theta}{2}=1-\frac{1}{r}\left(=: \frac{1}{r^{\prime}}\right) .
$$

Now, we interpolate (3.4) and (3.5) to obtain (3.3). More precisely, we follow the argument in [1, p. 1203]: For brevity, we set $u:=u_{N_{1}, \lambda_{1}}, v:=v_{N_{2}, \lambda_{2}}$. Then, [8, Lemma 6.4] implies that there exist decompositions $u=\sum_{k=1}^{\infty} u_{k}$, such that $\widehat{u_{k}} \subset \operatorname{supp} \widehat{u}$, and for any $q \geq 2$ we have $\left\|u_{k}\right\|_{U_{S}^{q}} \lesssim 2^{k\left(\frac{2}{q}-1\right)}\|u\|_{V_{S}^{2}}$, and the analogous decomposition for $v$. Then, by convexity, we obtain

$$
\begin{aligned}
\left\|P_{N_{3}} Q_{\lambda_{3}}(u v)\right\|_{L_{t}^{q^{\prime}} L_{x^{\prime}}^{r^{\prime}} L_{x_{1}}^{2}} & \lesssim \sum_{k, k^{\prime} \in \mathbb{N}}\left\|P_{N_{3}} Q_{\lambda_{3}}\left(u_{k} v_{k^{\prime}}\right)\right\|_{L_{t}^{q^{\prime}} L_{x^{\prime}}^{r^{\prime}} L_{x_{1}}^{2}} \\
& \lesssim \sum_{k, k^{\prime} \in \mathbb{N}}\left\|P_{N_{3}} Q_{\lambda_{3}}\left(u_{k} v_{k^{\prime}}\right)\right\|_{L_{t}^{\alpha} L_{x^{\prime}}^{\beta} L_{x_{1}}^{2}}^{\theta}\left\|P_{N_{3}} Q_{\lambda_{3}}\left(u_{k} v_{k^{\prime}}\right)\right\|_{L_{t, x}^{2}}^{1-\theta} .
\end{aligned}
$$

Estimates (3.4) and (3.5) further imply

$\left\|P_{N_{3}} Q_{\lambda_{3}}(u v)\right\|_{L_{t}^{q^{\prime}}} L_{x^{\prime}}^{r^{\prime}} L_{x_{1}}^{2} \lesssim\left(\sum_{k, k^{\prime} \in \mathbb{N}} 2^{k \theta\left(\frac{2}{q_{1}}-1\right)} 2^{k^{\prime} \theta\left(\frac{2}{q_{2}}-1\right)}\right) \frac{\lambda_{\min }^{\frac{\theta}{2}-\frac{\theta}{q_{2}}} N_{\min }^{\frac{d-1}{2}(1-\theta)}}{\lambda_{\max }^{\frac{\theta}{q_{1}}+\frac{1-\theta}{2}} N_{\max }^{\frac{1-\theta}{2}}}\|u\|_{V_{S}^{2}}\|v\|_{V_{S}^{2}}$.

Since $q_{1}, q_{2}>2$, the sums converge, and the proof of (3.3) is complete.

Define

$$
\mathcal{R}_{\lambda_{\max }, N_{\max }}=\left\{\left(\xi_{1}, \xi^{\prime}\right) \in \mathbb{R} \times \mathbb{R}^{d-1}|| \xi_{1}\left|\ll \lambda_{\max },\right| \xi^{\prime} \mid \ll N_{\max }\right\} .
$$


Lemma 3.3. Assume that there exist $\gamma_{1}, \gamma_{2}, \gamma_{3} \in \mathbb{R}^{d}$ such that $\gamma_{1}+\gamma_{2}-\gamma_{3} \in$ $\mathcal{R}_{4 \lambda_{\max }, 4 N_{\max }}$ and

$$
\operatorname{supp}_{\xi} \widehat{u}_{N_{i}, \lambda_{i}} \subset \mathcal{R}_{\lambda_{\max }, N_{\max }}+\gamma_{i}:=\left\{\xi \in \mathbb{R}^{d} \mid \xi-\gamma_{i} \in \mathcal{R}_{\lambda_{\max }, N_{\max }}\right\}
$$

Then, we have either

$$
|\nabla \varphi(\xi)-\nabla \varphi(\eta)| \gtrsim \lambda_{\max } N_{\max }
$$

for all $\xi \in \operatorname{supp}_{\xi} \widehat{u}_{N_{1}, \lambda_{1}}, \eta \in \operatorname{supp}_{\xi} \widehat{u}_{N_{2}, \lambda_{2}}$, or

$$
|\nabla \varphi(\eta)-\nabla \varphi(\zeta)| \gtrsim \lambda_{\max } N_{\max }
$$

for all $\eta \in \operatorname{supp}_{\xi} \widehat{u}_{N_{2}, \lambda_{2}}, \zeta \in \operatorname{supp}_{\xi} \widehat{u}_{N_{3}, \lambda_{3}}$.

Proof. Firstly, we consider the case $\max \left(\left|\xi^{\prime}\right|,\left|\eta^{\prime}\right|,\left|\zeta^{\prime}\right|\right) \ll N_{\max }$. We deduce from $\partial_{1} \varphi(\xi)=3 \xi_{1}^{2}+\left|\xi^{\prime}\right|^{2}$ that

$$
\begin{aligned}
& \left|\partial_{1} \varphi(\xi)-\partial_{1} \varphi(\eta)\right|+\left|\partial_{1} \varphi(\eta)-\partial_{1} \varphi(\xi+\eta)\right| \\
& \quad \geq 3\left|\xi_{1}^{2}-\eta_{1}^{2}\right|+3\left|\xi_{1}\left(\xi_{1}+2 \eta_{1}\right)\right|-\left|\xi^{\prime}\right|^{2}-\left|\eta^{\prime}\right|^{2}-\left|\xi^{\prime}+\eta^{\prime}\right|^{2} \gtrsim N_{\max }^{2},
\end{aligned}
$$

which implies the claim since $\left|\nabla^{2} \varphi(\xi)\right| \lesssim|\xi|$.

Next, we assume $\max \left(\left|\xi^{\prime}\right|,\left|\eta^{\prime}\right|,\left|\zeta^{\prime}\right|\right) \sim N_{\text {max }}$. For all $\xi \in \operatorname{supp}_{\xi} \widehat{u}_{N_{1}, \lambda_{1}}, \eta \in$ $\operatorname{supp}_{\xi} \widehat{u}_{N_{2}, \lambda_{2}}, \xi+\eta \in \operatorname{supp}_{\xi} \widehat{u}_{N_{3}, \lambda_{3}}$, we will show

$$
\sum_{j=2}^{d}\left(\left|\partial_{j} \varphi(\xi)-\partial_{j} \varphi(\eta)\right|+\left|\partial_{j} \varphi(\eta)-\partial_{j} \varphi(\xi+\eta)\right|\right) \gtrsim \lambda_{\max } N_{\max }
$$

We may assume $\left|\xi^{\prime}\right| \sim N_{\max }, \lambda_{1} \sim \lambda_{\max }$. For $2 \leq j \leq d$, it is observed that $\partial_{j} \varphi(\xi)=2 \xi_{1} \xi_{j}$. Then, for (3.9), it suffices to show

$$
\left|\xi_{1} \xi^{\prime}-\eta_{1} \eta^{\prime}\right|+\left|\eta_{1} \eta^{\prime}-\left(\xi_{1}+\eta_{1}\right)\left(\xi^{\prime}+\eta^{\prime}\right)\right| \gtrsim \lambda_{1} N_{1} .
$$

Since $\left|\xi^{\prime}\right| \sim N_{\max }, \lambda_{1} \sim \lambda_{\max }$, if either $\lambda_{\min } \ll \lambda_{\max }$ or $\min \left(\left|\eta^{\prime}\right|,\left|\xi^{\prime}+\eta^{\prime}\right|\right) \ll N_{\max }$ holds, we easily verify (3.10). Then, we assume $\lambda_{1} \sim \lambda_{2} \sim \lambda_{3}$ and $\left|\xi^{\prime}\right| \sim\left|\eta^{\prime}\right| \sim$ $\left|\xi^{\prime}+\eta^{\prime}\right|$. We observe

$$
\begin{aligned}
& \left|\eta_{1} \eta^{\prime}-\left(\xi_{1}+\eta_{1}\right)\left(\xi^{\prime}+\eta^{\prime}\right)\right| \\
& \quad=\left|\eta_{1} \eta^{\prime}-\left(\xi_{1}+\eta_{1}\right)\left(\xi^{\prime}-\frac{\eta_{1}}{\xi_{1}} \eta^{\prime}+\frac{\eta_{1}}{\xi_{1}} \eta^{\prime}+\eta^{\prime}\right)\right| \\
& \quad \geq\left|\eta_{1} \eta^{\prime}-\left(\xi_{1}+\eta_{1}\right)\left(1+\frac{\eta_{1}}{\xi_{1}}\right) \eta^{\prime}\right|-\left|\left(1+\frac{\eta_{1}}{\xi_{1}}\right)\left(\xi_{1} \xi^{\prime}-\eta_{1} \eta^{\prime}\right)\right| \\
& \quad=\left|1+\frac{\eta_{1}}{\xi_{1}}+\frac{\xi_{1}}{\eta_{1}}\right|\left|\eta_{1} \eta^{\prime}\right|-\left|\left(1+\frac{\eta_{1}}{\xi_{1}}\right)\left(\xi_{1} \xi^{\prime}-\eta_{1} \eta^{\prime}\right)\right| .
\end{aligned}
$$

Since $\left|\alpha+\alpha^{-1}\right| \geq 2$ for any $\alpha \in \mathbb{R}$, this completes the proof of (3.10). 
From (3.9), without loss of generality, we can assume that there exist $\xi_{0} \in$ $\operatorname{supp}_{\xi} \widehat{u}_{N_{1}, \lambda_{1}}, \eta_{0} \in \operatorname{supp}_{\xi} \widehat{u}_{N_{2}, \lambda_{2}}$ such that

$$
\sum_{j=2}^{d}\left|\partial_{j} \varphi\left(\xi_{0}\right)-\partial_{j} \varphi\left(\eta_{0}\right)\right| \gtrsim \lambda_{\max } N_{\max }
$$

For $2 \leq j, k \leq d$ and all $\xi \in \operatorname{supp}_{\xi} \widehat{u}_{N_{1}, \lambda_{1}}, \eta \in \operatorname{supp}_{\xi} \widehat{u}_{N_{2}, \lambda_{2}}$, since $\left|\partial_{1} \partial_{j} \varphi(\xi)\right|+$ $\left|\partial_{1} \partial_{j} \varphi(\eta)\right| \lesssim N_{\max }$ and $\left|\partial_{k} \partial_{j} \varphi(\xi)\right|+\left|\partial_{k} \partial_{j} \varphi(\eta)\right| \lesssim \lambda_{\max }$, we get

$$
\left|\partial_{j} \varphi(\xi)-\partial_{j} \varphi\left(\xi_{0}\right)\right|+\left|\partial_{j} \varphi(\eta)-\partial_{j} \varphi\left(\eta_{0}\right)\right| \ll \lambda_{\max } N_{\max },
$$

for all $\xi \in \operatorname{supp}_{\xi} \widehat{u}_{N_{1}, \lambda_{1}}, \eta \in \operatorname{supp}_{\xi} \widehat{u}_{N_{2}, \lambda_{2}}$. This estimate and (3.11) yield the claim (3.7).

Now, we define the solution spaces as $Y^{s}:=C\left(\mathbb{R} ; H^{s}\left(\mathbb{R}^{d}\right)\right) \cap\left\langle\nabla_{x}\right\rangle^{-s} V_{S}^{2}$ and $\dot{Y}^{s}:=C\left(\mathbb{R} ; \dot{H}^{s}\left(\mathbb{R}^{d}\right)\right) \cap\left|\nabla_{x}\right|^{-s} V_{S}^{2}$, with norms

$$
\begin{aligned}
\|u\|_{Y^{s}} & :=\left(\sum_{N \in 2^{\mathbb{Z}}}\langle N\rangle^{2 s}\left\|P_{N} u\right\|_{V_{S}^{2}}^{2}\right)^{1 / 2}, \\
\|u\|_{\dot{Y}^{s}} & :=\left(\sum_{N \in 2^{\mathbb{Z}}} N^{2 s}\left\|P_{N} u\right\|_{V_{S}^{2}}^{2}\right)^{1 / 2},
\end{aligned}
$$

respectively.

Proposition 3.4. Let $d \geq 6$. Then, we have

$$
\left\|\mathcal{I}\left(\partial_{x_{1}}\left(u_{1} u_{2}\right)\right)\right\|_{Y^{s_{C}}} \lesssim\left\|u_{1}\right\|_{Y^{s_{c}}}\left\|u_{2}\right\|_{Y^{s_{C}}}, \quad\left\|\mathcal{I}\left(\partial_{x_{1}}\left(u_{1} u_{2}\right)\right)\right\|_{\dot{Y}^{s_{C}}} \lesssim\left\|u_{1}\right\|_{\dot{Y}^{s_{c}}}\left\|u_{2}\right\|_{\dot{Y}^{s_{c}}} .
$$

Proof. We show first that there exists $\varepsilon>0$ such that for any $N_{1}, N_{2}, N_{3} \in 2^{\mathbb{Z}}$ we have

$$
\left|\iint P_{N_{1}} u_{1} P_{N_{2}} u_{2} \partial_{x_{1}} P_{N_{3}} u_{3} d x d t\right| \lesssim N_{\min }^{s_{c}+\varepsilon} N_{\max }^{-\varepsilon} \prod_{i=1}^{3}\left\|P_{N_{i}} u_{i}\right\|_{V_{S}^{2}} .
$$

As before, we use the shorthand notation $u_{N_{j}}:=P_{N_{j}} u_{j}, u_{N_{j}, \lambda_{j}}:=Q_{\lambda_{j}} P_{N_{j}} u_{j}$, etc. Obviously, (3.12) is implied by

$$
\sum_{\lambda_{1}, \lambda_{2}, \lambda_{3} \in 2^{\mathbb{Z}}} \lambda_{3}\left|\iint u_{N_{1}, \lambda_{1}} u_{N_{2}, \lambda_{2}} u_{N_{3}, \lambda_{3}} d x d t\right| \lesssim N_{\min }^{s_{c}+\varepsilon} N_{\max }^{-\varepsilon} \prod_{i=1}^{3}\left\|u_{N_{i}}\right\|_{V_{S}^{2}}
$$

Now we show (3.13). After harmless decompositions, we may assume that there exist $\gamma_{1}, \gamma_{2}, \gamma_{3} \in \mathbb{R}^{d}$ such that $\gamma_{1}+\gamma_{2}-\gamma_{3} \in \mathcal{R}_{4 \lambda_{\max }, 4 N_{\max }}$ and (3.6). Lemma 3.3 provides either $|\nabla \varphi(\xi)-\nabla \varphi(\eta)| \gtrsim \lambda_{\max } N_{\max }$ for all $\xi \in \operatorname{supp}_{\xi} u_{N_{1}, \lambda_{1}}, \eta \in$ $\operatorname{supp}_{\xi} u_{N_{2}, \lambda_{2}}$ or $|\nabla \varphi(\eta)-\nabla \varphi(\zeta)| \gtrsim \lambda_{\max } N_{\max }$ for all $\eta \in \operatorname{supp}_{\xi} u_{N_{2}, \lambda_{2}}$ and $\zeta \in$ 
$\operatorname{supp}_{\xi} u_{N_{3}, \lambda_{3}}$. For the former case, it follows from the Hölder's inequality, the Strichartz estimate (3.2), and the bilinear estimate (3.3) that

$$
\begin{aligned}
& \quad \sum_{\lambda_{1}, \lambda_{2}, \lambda_{3} \in 2^{\mathbb{Z}}} \lambda_{3}\left|\iint u_{N_{1}, \lambda_{1}} u_{N_{2}, \lambda_{2}} u_{N_{3}, \lambda_{3}} d x d t\right| \\
& \leq \sum_{\lambda_{i} \leq N_{i}(i=1,2,3)} \lambda_{3}\left\|P_{N_{3}} Q_{\lambda_{3}}\left(u_{N_{1}, \lambda_{1}} u_{N_{2}, \lambda_{2}}\right)\right\|_{L_{t}^{q^{\prime}} L_{x^{\prime}}^{r^{\prime}} L_{x_{1}}^{2}}\left\|u_{N_{3}, \lambda_{3}}\right\|_{L_{t}^{q} L_{x^{\prime}}^{r} L_{x_{1}}^{2}} \\
& \leq \sum_{\lambda_{i} \leq N_{i}(i=1,2,3)} \lambda_{\max }^{\frac{d-1}{d-3} \varepsilon} \lambda_{\min }^{\frac{1}{d-3}-\frac{d-1}{d-3} \varepsilon} N_{\max }^{-\frac{1}{2}+\frac{1}{d-3}} N_{\min }^{\frac{d-3}{2}-\frac{2}{d-3}}\left\|u_{N_{1}, \lambda_{1}}\right\|_{V_{S}^{2}}\left\|u_{N_{2}, \lambda_{2}}\right\|_{V_{S}^{2}}\left\|u_{N_{3}, \lambda_{3}}\right\|_{V_{S}^{2}} \\
& \leq N_{\min }^{s_{c}+\frac{1}{2}-\frac{1}{d-3}-\frac{d-1}{d-3} \varepsilon} N_{\max }^{-\frac{1}{2}+\frac{1}{d-3}+\frac{d-1}{d-3} \varepsilon}\left\|u_{N_{1}}\right\|_{V_{S}^{2}}\left\|u_{N_{2}}\right\|_{V_{S}^{2}}\left\|u_{N_{3}}\right\|_{V_{S}^{2}} .
\end{aligned}
$$

Here, the pair $(q, r)$ should satisfy the hypothesis of Proposition 3.2, and we have used $\lambda_{\max } \leq N_{\max }$ and $\lambda_{\min } \leq N_{\min }$. In the similar way, the latter case is treated as follows:

$$
\begin{aligned}
& \sum_{\lambda_{1}, \lambda_{2}, \lambda_{3} \in 2^{\mathbb{Z}}} \lambda_{3}\left|\iint u_{N_{1}, \lambda_{1}} u_{N_{2}, \lambda_{2}} u_{N_{3}, \lambda_{3}} d x d t\right| \\
\leq & \sum_{\lambda_{i} \leq N_{i}(i=1,2,3)} \lambda_{3}\left\|P_{N_{1}} Q_{\lambda_{1}}\left(u_{N_{2}, \lambda_{2}} u_{N_{3}, \lambda_{3}}\right)\right\|_{L_{t}^{q^{\prime}} L_{x^{\prime}}^{r^{\prime}} L_{x_{1}}^{2}}\left\|u_{N_{1}, \lambda_{1}}\right\|_{L_{t}^{q} L_{x^{\prime}}^{r^{\prime} L_{x_{1}}^{2}}} \\
\leq & N_{\min }^{s_{c}+\frac{1}{2}-\frac{1}{d-3}-\frac{d-1}{d-3} \varepsilon} N_{\max }^{-\frac{1}{2}+\frac{1}{d-3}+\frac{d-1}{d-3} \varepsilon}\left\|u_{N_{1}}\right\|_{V_{S}^{2}}\left\|u_{N_{2}}\right\|_{V_{S}^{2}}\left\|u_{N_{3}}\right\|_{V_{S}^{2}} .
\end{aligned}
$$

Finally, we explain why (3.12) implies Proposition 3.4. By duality, see, e.g., [1, Lemma 7.3], we obtain

$$
\left\|P_{N_{3}} \mathcal{I}\left(\partial_{x_{1}}\left(P_{N_{1}} u_{1} P_{N_{2}} u_{2}\right)\right)\right\|_{V_{S}^{2}} \lesssim N_{\min }^{s_{c}}\left(\frac{N_{\min }}{N_{\max }}\right)^{\varepsilon}\left\|P_{N_{1}} u_{1}\right\|_{V_{S}^{2}}\left\|P_{N_{2}} u_{2}\right\|_{V_{S}^{2}} .
$$

This can be easily summed up.

Again, the proof of Theorem 1.2 is a straightforward application of the contraction mapping principle. The scattering claim follows from the well-known fact that functions in $V^{2}$ have limits at $\pm \infty$.

\section{Radial Strichartz estimates and the proof of Theorem $\mathbf{1 . 3}$}

We first prove a variant of the Strichartz estimates in 2.1 for functions which, for fixed $x_{1}$, are radial in $x^{\prime}$.

Theorem 4.1. Let $d \geq 3$ and $2 \leq q, r \leq \infty$ satisfy

$$
\begin{aligned}
& \frac{2}{q} \leq(2 d-3)\left(\frac{1}{2}-\frac{1}{r}\right), \quad(d, q, r) \neq(3,2, \infty), \quad(q, r) \neq\left(2, \frac{2(2 d-3)}{2 d-5}\right), \\
& \text { and let } \sigma=-\frac{d-1}{2}+\frac{d-1}{r}+\frac{2}{q} \text {. Then, for all functions } f \in L_{\mathrm{rad}}^{2}\left(\mathbb{R}^{d}\right), \text { we have } \\
& \left\|D_{x_{1}}^{\frac{1}{q}}\left|\nabla_{x^{\prime}}\right|^{\sigma} e^{t S} f\right\|_{L_{t}^{q} L_{x^{\prime}}^{r} L_{x_{1}}^{2}} \lesssim\|f\|_{L_{x}^{2}} .
\end{aligned}
$$


The proof follows the exact same lines as the proof of Theorem 2.1, but with the Strichartz estimates for the $(d-1)$-dimensional Schrödinger equation from [6] replaced by the radial version obtained in [2, Theorem 1.1].

Lemma 4.2. Let $d \geq 2$ and $f_{N_{1}, \lambda_{1}, M_{1}}=R_{M_{1}} Q_{\lambda_{1}} P_{N_{1}} f, g_{N_{2}, \lambda_{2}, M_{2}}=R_{M_{2}} Q_{\lambda_{2}} P_{N_{2}} g$. (i) Suppose that there exists $\ell \in\{2, \ldots, d\}$ such that

$$
\left|\partial_{\ell} \varphi(\xi)-\partial_{\ell} \varphi(\eta)\right| \gtrsim N_{\max }^{2}
$$

for all $\xi \in \operatorname{supp} \widehat{f}_{N_{1}, \lambda_{1}, M_{1}}, \eta \in \operatorname{supp} \widehat{g}_{N_{2}, \lambda_{2}, M_{2}}$. Then, it holds that

$$
\begin{aligned}
& \left\|P_{N_{3}}\left(e^{t S} f_{\lambda_{1}, N_{1}, M_{1}} e^{t S} g_{\lambda_{2}, N_{2}, M_{2}}\right)\right\|_{L_{t}^{2} L_{x}^{2}} \\
& \quad \lesssim\left(\frac{\min \left\{\lambda_{1}, \lambda_{2}\right\} \min \left\{M_{1}, M_{2}\right\}^{d-2}}{N_{\max }^{2}}\right)^{\frac{1}{2}}\left\|f_{M_{1}, \lambda_{1}, N_{1}}\right\|_{L^{2}}\left\|g_{M_{2}, \lambda_{2}, N_{2}}\right\|_{L^{2}}
\end{aligned}
$$

(ii) Suppose that

$$
\left|\partial_{1} \varphi(\xi)-\partial_{1} \varphi(\eta)\right| \gtrsim N_{\max }^{2}
$$

for all $\xi \in \operatorname{supp} \widehat{f}_{N_{1}, \lambda_{1}, M_{1}}, \eta \in \operatorname{supp} \widehat{g}_{N_{2}, \lambda_{2}, M_{2}}$. Then, it holds that

$$
\begin{aligned}
& \left\|P_{N_{3}}\left(e^{t S} f_{\lambda_{1}, N_{1}, M_{1}} e^{t S} g_{\lambda_{2}, N_{2}, M_{2}}\right)\right\|_{L_{t}^{2} L_{x}^{2}} \\
& \quad \lesssim\left(\frac{\min \left\{M_{1}, M_{2}\right\}^{d-1}}{N_{\max }^{2}}\right)^{\frac{1}{2}}\left\|f_{M_{1}, \lambda_{1}, N_{1}}\right\|_{L^{2}}\left\|g_{M_{2}, \lambda_{2}, N_{2}}\right\|_{L^{2}} .
\end{aligned}
$$

As above, the proof of this lemma follows from [1, Lemma 2.6]. As above, it immediately extends to $U_{S}^{2}$-functions.

Let $Y_{\text {rad }}^{s}$ and $\dot{Y}_{\text {rad }}^{s}$ be the subspaces of $Y^{s}$ and $\dot{Y}^{s}$ of functions which, for fixed $x_{1}$, are radial in $x^{\prime}$, with the same norms. Then, the key for the proof of Theorem 1.3 is the following

Proposition 4.3. Let $d \geq 4$. Then, we have

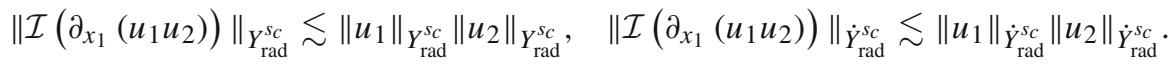

Proof. For $i=1,2,3$, we use $u_{i}:=R_{M_{i}} Q_{\lambda_{i}} P_{N_{i}} u$. As in the proof of Proposition 3.4, it suffices to show

$$
\sum_{\lambda_{i}, M_{i}} \lambda_{\max }\left|\iint u_{1} u_{2} u_{3} d x d t\right| \lesssim N_{\min }^{s_{c}+\varepsilon} N_{\max }^{-\varepsilon} \prod_{i=1}^{3}\left\|u_{N_{i}}\right\|_{V_{S}^{2}}
$$


Here and in the sequel, all functions are implicitly assumed to satisfy the radiality hypothesis. Let

$$
\begin{aligned}
& \left(\frac{1}{q_{1}}, \frac{1}{r_{1}}\right)=\left(\frac{1}{2}-\varepsilon, \frac{2 d-5}{2(2 d-3)}\right), \\
& \left(\frac{1}{q_{2}}, \frac{1}{r_{2}}\right)=\left(\frac{(d-1)(2 d-3)}{2(d-1+2(2 d-3) \varepsilon)} \varepsilon, \frac{d-1}{2(d-1+2(2 d-3) \varepsilon)}\right), \\
& \left(\frac{1}{q_{3}}, \frac{1}{r_{3}}\right)=\left(2 \varepsilon, \frac{2}{2 d-3}\right) .
\end{aligned}
$$

Then, we have

$$
\begin{aligned}
& \left\|R_{M} Q_{\lambda} P_{N} u\right\|_{L_{t}^{q_{1}} L_{x^{\prime}}^{r_{1}} L_{x_{1}}^{2}} \lesssim \lambda^{-\frac{1}{q_{1}}} M^{-\frac{d-2}{2 d-3}+2 \varepsilon}\left\|R_{M} Q_{\lambda} P_{N} u\right\|_{U_{S}^{q_{1}}}, \\
& \left\|R_{M} Q_{\lambda} P_{N} u\right\|_{L_{t}^{q_{2}} L_{x^{\prime}}^{r_{2}} L_{x_{1}}^{2}} \lesssim \lambda^{-\frac{1}{q_{2}}}\left\|R_{M} Q_{\lambda} P_{N} u\right\|_{U_{S}^{q_{2}}}, \\
& \left\|R_{M} Q_{\lambda} P_{N} u\right\|_{L_{t}^{q_{3}} L_{x^{\prime}}^{r_{3}} L_{x_{1}}^{2}} \lesssim \lambda^{-\frac{1}{q_{3}}} M^{\frac{(d-1)(2 d-7)}{2(2 d-3)}-4 \varepsilon}\left\|R_{M} Q_{\lambda} P_{N} u\right\|_{U_{S}^{q_{3}}} .
\end{aligned}
$$

By symmetry of (4.4), we may assume $N_{3} \lesssim N_{1} \sim N_{2}, \lambda_{2} \lesssim \lambda_{1}$, and then, it is enough to consider the following three cases:

(1) $M_{1} \sim N_{1}, M_{2} \sim N_{2}$. (2) $M_{1} \sim N_{1}, M_{2} \ll N_{1}$, (3) $M_{1} \ll N_{1}, M_{2} \ll N_{1}$.

(1) First, we assume $M_{1} \sim N_{1}, M_{2} \sim N_{2}$. By using (4.5) and (4.7), we obtain

$$
\begin{aligned}
& \left|\iint u_{1} u_{2} u_{3} d x d t\right| \\
& \lesssim \lambda_{\min }^{\frac{1}{2}}\left\|u_{1}\right\|_{L_{t}^{q_{1}} L_{x^{\prime}}^{r_{1}} L_{x_{1}}^{2}}^{2}\left\|u_{2}\right\|_{L_{t}^{q_{1}} L_{x^{\prime}}^{r_{1}} L_{x_{1}}^{2}}\left\|u_{3}\right\|_{L_{t}^{q_{3}} L_{x^{\prime}}^{r_{3}} L_{x_{1}}^{2}} \\
& \lesssim \lambda_{\min }^{\frac{1}{2}} \lambda_{1}^{-\frac{1}{q_{1}}} \lambda_{2}^{-\frac{1}{q_{1}}} \lambda_{3}^{-2 \varepsilon} M_{3}^{\frac{(d-1)(2 d-7)}{2(2 d-3)}-4 \varepsilon} N_{1}^{-\frac{2(d-2)}{2 d-3}+4 \varepsilon} \prod_{i=1,2,3}\left\|u_{i}\right\|_{V_{S}^{2}} \\
& \lesssim \lambda_{\min }^{\varepsilon} \lambda_{\max }^{-1} M_{3}^{s_{c}+\varepsilon} N_{1}^{-2 \varepsilon} \prod_{i=1,2,3}\left\|u_{i}\right\|_{V_{S}^{2}}
\end{aligned}
$$

which completes (4.4).

(2) In the case $M_{1} \sim N_{1}, M_{2} \ll N_{1}$, it is observed that $\lambda_{2} \sim M_{3} \sim N_{1}$. Then, without loss of generality, we may assume $\lambda_{3} \lesssim \lambda_{1} \sim N_{1}$. In the case $\lambda_{3} \ll \lambda_{1}$, for all $\xi \in \operatorname{supp}_{\xi} \widehat{u}_{1}, \eta \in \operatorname{supp}_{\xi} \widehat{u}_{2}$ such that $\xi+\eta \in \operatorname{supp}_{\xi} \widehat{u}_{3}$, we observe

$$
\begin{aligned}
& \left|\tau_{1}-\varphi(\xi)\right|+\left|\tau_{2}-\varphi(\eta)\right|+\left|\tau_{1}+\tau_{2}-\varphi(\xi+\eta)\right| \\
& \quad \gtrsim|\varphi(\xi+\eta)-\varphi(\xi)-\varphi(\eta)| \\
& \left.\quad \gtrsim\left|\xi_{1}\right| \xi^{\prime}\right|^{2}|-| \xi_{1}+\eta_{1}|||\xi+\eta|^{2}+\xi_{1}^{2}-\xi_{1} \eta_{1}+\eta_{1}^{2}|-| \eta_{1}\left|\eta^{\prime}\right|^{2} \mid \gtrsim N_{1}^{3} .
\end{aligned}
$$

Thus, we can assume that at least one of $u_{1}, u_{2}, u_{3}$ satisfies supp $\widehat{u}_{i} \subset$ $\left\{(\tau, \xi)|| \tau-\varphi(\xi) \mid \gtrsim N_{1}^{3}\right\}$. We easily see that this condition verifies the claim by 
utilizing Theorem 2.1 and (3.2). For example, if supp $\widehat{u}_{1} \subset\{(\tau, \xi)|| \tau-\varphi(\xi) \mid \gtrsim$ $\left.N_{1}^{3}\right\}$, using Bernstein's inequality and Theorem 4.1 we obtain

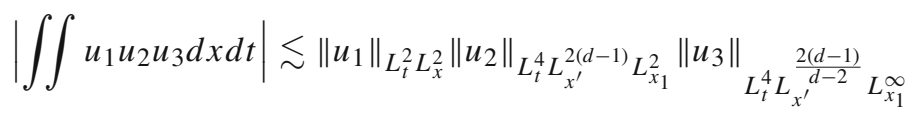

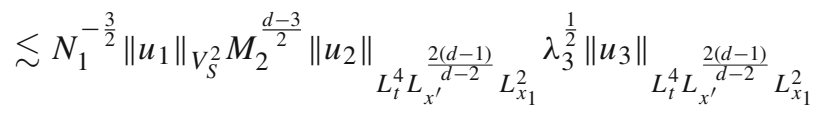

$$
\begin{aligned}
& \lesssim \lambda_{3}^{\frac{1}{4}} M_{2}^{s_{c}+\frac{1}{2}} N_{1}^{-\frac{7}{4}} \prod_{i=1,2,3}\left\|u_{i}\right\|_{V_{S}^{2}}
\end{aligned}
$$

Next we consider the case $\lambda_{1} \sim \lambda_{2} \sim \lambda_{3} \sim N_{1}$. Since $M_{2} \ll M_{1} \sim \lambda_{1}$, we may assume that there exists $\ell \in\{2, \ldots, d\}$ such that $\left|\partial_{\ell} \varphi(\xi)-\partial_{\ell} \varphi(\eta)\right| \gtrsim N_{1}^{2}$ for $\xi \in \operatorname{supp}_{\xi} \widehat{u}_{1}, \eta \in \operatorname{supp}_{\xi} \widehat{u}_{2}$. Then, from (4.2) we get

$$
\left\|u_{1} u_{2}\right\|_{L_{t}^{2} L_{x}^{2}} \lesssim M_{2}^{\frac{d-2}{2}} N_{1}^{-\frac{1}{2}}\left\|u_{1}\right\|_{U_{S}^{2}}\left\|u_{2}\right\|_{U_{S}^{2}}
$$

On the other hand, for $\left(\frac{1}{\alpha}, \frac{1}{\beta}\right)=\left(\frac{1}{q_{1}}+\frac{1}{q_{2}}, \frac{1}{r_{1}}+\frac{1}{r_{2}}\right)$, we have

$$
\begin{aligned}
\left\|u_{1} u_{2}\right\|_{L_{t}^{\alpha} L_{x^{\prime}}^{\beta} L_{x_{1}}^{2}} & \lesssim \lambda_{1}^{\frac{1}{2}}\left\|u_{1}\right\|_{L_{t}^{q_{1}} L_{x^{\prime}}^{r_{1}} L_{x_{1}}^{2}\left\|u_{2}\right\|_{L_{t}^{q_{2}} L_{x^{\prime}}^{r_{2}} L_{x_{1}}^{2}}} \\
& \lesssim \lambda_{1}^{\frac{1}{2}-\frac{1}{q_{1}}-\frac{1}{q_{2}}} N_{1}^{-\frac{d-2}{2 d-3}+2 \varepsilon}\left\|u_{1}\right\|_{U_{S}^{q_{1}}}\left\|u_{2}\right\|_{U_{S}^{q_{2}}} \\
& \sim N_{1}^{-\frac{1}{q_{2}}-\frac{d-2}{2 d-3}+3 \varepsilon}\left\|u_{1}\right\|_{U_{S}^{q_{1}}}\left\|u_{2}\right\|_{U_{S}^{q_{2}}} .
\end{aligned}
$$

We notice that $\alpha, \beta \geq 1$ and $q_{1}, q_{2}>2$ if $\varepsilon>0$ is chosen sufficiently small. Let $\theta=\frac{2(d-1)+4(2 d-3) \varepsilon}{(d-1)(2 d-5)-4(2 d-3) \varepsilon}$. Then, since

$$
\frac{\theta}{\alpha}+\frac{1-\theta}{2}=\frac{1}{q_{1}^{\prime}}, \quad \frac{\theta}{\beta}+\frac{1-\theta}{2}=\frac{1}{r_{1}^{\prime}}
$$

by interpolating the above two estimates (with a similar argument as in the proof of Proposition 3.4), we have

$$
\left\|u_{1} u_{2}\right\|_{L_{t}^{q_{1}^{\prime}} L_{x^{\prime}}^{r_{1}^{\prime}} L_{x_{1}}^{2}} \lesssim M_{2}^{\frac{d-2}{2}(1-\theta)} N_{1}^{-\frac{1}{2}-\frac{\theta}{q_{2}}+\frac{\theta}{2(2 d-3)}+3 \varepsilon \theta}\left\|u_{1}\right\|_{V_{S}^{2}}\left\|u_{2}\right\|_{V_{S}^{2}}
$$

This and (4.5) yield

$$
\begin{aligned}
\left|\iint u_{1} u_{2} u_{3} d x d t\right| & \lesssim\left\|u_{1} u_{2}\right\|_{L_{t}^{q_{1}^{\prime}} L_{x^{\prime}}^{r_{1}^{\prime}} L_{x_{1}}^{2}}\left\|u_{3}\right\|_{L_{t}^{q_{1}} L_{x^{\prime}}^{r_{1}} L_{x_{1}}^{2}} \\
& \lesssim M_{2}^{\frac{d-2}{2}(1-\theta)} N_{1}^{-\frac{3 d-5}{2 d-3}-\frac{\theta}{q_{2}}+\frac{\theta}{2(2 d-3)}+3 \varepsilon(1+\theta)} \prod_{i=1,2,3}\left\|u_{i}\right\|_{V_{S}^{2}} \\
& \lesssim M_{2}^{s_{c}+\varepsilon} N_{1}^{-1-\varepsilon} \prod_{i=1,2,3}\left\|u_{i}\right\|_{V_{S}^{2}}
\end{aligned}
$$


(3) We deal with the last case $M_{1} \ll N_{1}, M_{2} \ll N_{1}$. By symmetry, we assume $M_{2} \leq$ $M_{1}$. Assume first that $M_{1} \gtrsim\left(\lambda_{3} N_{1}\right)^{\frac{1}{2}}$ which implies $\lambda_{3} \ll \lambda_{1} \sim \lambda_{2} \sim N_{1}$. Thus, we observe that $\left|\partial_{1} \varphi(\xi)-\partial_{1} \varphi(\eta)\right| \gtrsim N_{1}^{2}$ for $\xi \in \operatorname{supp}_{\xi} \widehat{u}_{2}, \eta \in \operatorname{supp}_{\xi} \widehat{u}_{3}$. (4.3) implies

$$
\left\|u_{2} u_{3}\right\|_{L_{t}^{2} L_{x}^{2}} \lesssim M_{\min }^{\frac{d-1}{2}} N_{1}^{-1}\left\|u_{2}\right\|_{U_{S}^{2}}\left\|u_{3}\right\|_{U_{S}^{2}}
$$

While, similarly to the above observation, we get

$$
\left\|u_{2} u_{3}\right\|_{L_{t}^{\alpha} L_{x^{\prime}}^{\beta} L_{x_{1}}^{2}} \lesssim \lambda_{2}^{-\frac{1}{q_{1}}} \lambda_{3}^{\frac{1}{2}-\frac{1}{q_{2}}} M_{\min }^{-\frac{d-2}{2 d-3}+2 \varepsilon}\left\|u_{2}\right\|_{U_{S}^{q_{1}}}\left\|u_{3}\right\|_{U_{S}^{q_{2}}}
$$

Interpolating the above two, we get

$\left\|u_{2} u_{3}\right\|_{L_{t}^{q_{1}^{\prime}} L_{x^{\prime}}^{r_{1}^{\prime}} L_{x_{1}}^{2}} \lesssim \lambda_{2}^{-\frac{\theta}{q_{1}}} \lambda_{3}^{\frac{\theta}{2}-\frac{\theta}{q_{2}}} M_{\min }^{\frac{d-1}{2}(1-\theta)-\frac{d-2}{2 d-3} \theta+2 \varepsilon \theta} N_{1}^{-1+\theta}\left\|u_{1}\right\|_{V_{S}^{2}}\left\|u_{2}\right\|_{V_{S}^{2}}$.

Consequently, it follows from $M_{1} \gtrsim \max \left\{M_{\min },\left(\lambda_{3} N_{1}\right)^{\frac{1}{2}}\right\}$ that

$$
\begin{aligned}
& \left|\iint u_{1} u_{2} u_{3} d x d t\right| \\
& \quad \lesssim\left\|u_{1}\right\|_{L_{t}^{q_{1}} L_{x^{\prime}}^{r_{1}} L_{x_{1}}^{2}}\left\|u_{2} u_{3}\right\|_{L_{t}^{q_{1}^{\prime}} L_{x^{\prime}}^{r_{1}^{\prime}} L_{x_{1}}^{2}} \\
& \quad \lesssim \lambda_{1}^{-\frac{1+\theta}{q_{1}}} \lambda_{3}^{\frac{\theta}{2}-\frac{\theta}{q_{2}}} M_{1}^{-\frac{d-2}{2 d-3}+2 \varepsilon} M_{\min }^{\frac{d-1}{2}(1-\theta)-\frac{d-2}{2 d-3} \theta+2 \varepsilon \theta} N_{1}^{-1+\theta} \prod_{i=1,2,3}\left\|u_{i}\right\|_{V_{S}^{2}} \\
& \quad \lesssim \lambda_{1}^{-1} \lambda_{3}^{\varepsilon} M_{\min }^{s_{c}+\varepsilon} N_{1}^{-2 \varepsilon} \prod_{i=1,2,3}\left\|u_{i}\right\|_{V_{S}^{2}} .
\end{aligned}
$$

In the case $M_{1} \ll\left(\lambda_{3} N_{1}\right)^{\frac{1}{2}}$, we easily observe that at least one of $u_{1}, u_{2}, u_{3}$ satisfies supp $\widehat{u}_{i} \subset\left\{(\tau, \xi)|| \tau-\varphi(\xi) \mid \gtrsim \lambda_{3} N_{1}^{2}\right\}$. Indeed, $M_{2} \lesssim M_{1} \ll\left(\lambda_{3} N_{1}\right)^{\frac{1}{2}}$ yields

$$
\begin{aligned}
& \left|\tau_{1}-\varphi(\xi)\right|+\left|\tau_{2}-\varphi(\eta)\right|+\left|\tau_{1}+\tau_{2}-\varphi(\xi+\eta)\right| \\
& \quad \geq|\varphi(\xi+\eta)-\varphi(\xi)-\varphi(\eta)| \\
& \quad \geq x 3\left|\xi_{1} \eta_{1}\left(\xi_{1}+\eta_{1}\right)\right|-10\left(\left|\xi_{1}\right|+\left|\eta_{1}\right|\right)\left(\left|\xi^{\prime}\right|^{2}+\left|\eta^{\prime}\right|^{2}\right) \gtrsim \lambda_{3} N_{1}^{2},
\end{aligned}
$$

for all $\xi \in \operatorname{supp}_{\xi} \widehat{u}_{1}, \eta \in \operatorname{supp}_{\xi} \widehat{u}_{2}$ which satisfy $\xi+\eta \in \operatorname{supp}_{\xi} \widehat{u}_{3}$. In the case supp $\widehat{u}_{1} \subset\left\{(\tau, \xi)|| \tau-\varphi(\xi) \mid \gtrsim \lambda_{3} N_{1}^{2}\right\}$ and $M_{2} \lesssim M_{3}$, since $M_{2} \lesssim M_{1} \ll$ $\left(\lambda_{3} N_{1}\right)^{\frac{1}{2}}$, it follows from the Strichartz estimates (3.2) and Bernstein's inequality 
that

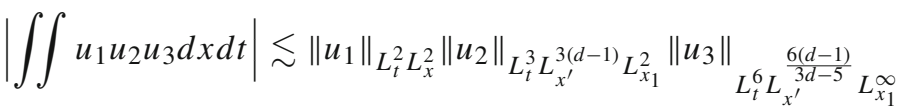

$$
\begin{aligned}
& \lesssim \lambda_{3}^{-\frac{1}{2}} N_{1}^{-1}\left\|u_{1}\right\|_{V_{S}^{2}} M_{2}^{\frac{d-3}{2}}\left\|u_{2}\right\|{ }_{L_{t}^{3} L_{x^{\prime}}^{\frac{6(d-1)}{3 d-7}}} L_{x_{1}}^{2} \lambda_{3}^{\frac{1}{2}}\left\|u_{3}\right\| L_{t}^{6} L_{x^{\prime}}^{\frac{6(d-1)}{3 d-5}} L_{x_{1}}^{2} \\
& \lesssim \lambda_{3}^{-\frac{1}{6}} M_{1}^{\frac{1}{3}+2 \varepsilon} M_{2}^{s_{c}+\frac{1}{6}-2 \varepsilon} N_{1}^{-\frac{4}{3}} \prod_{i=1,2,3}\left\|u_{i}\right\|_{V_{S}^{2}} \\
& \lesssim \lambda_{3}^{\varepsilon} M_{2}^{s_{c}+\frac{1}{6}-2 \varepsilon} N_{1}^{-\frac{7}{6}+\varepsilon} \prod_{i=1,2,3}\left\|u_{i}\right\|_{V_{S}^{2}}
\end{aligned}
$$

The other cases are treated similarly.

As above, Theorem 1.3 follows by the standard argument.

Funding Open Access funding enabled and organized by Projekt DEAL.

Open Access. This article is licensed under a Creative Commons Attribution 4.0 International License, which permits use, sharing, adaptation, distribution and reproduction in any medium or format, as long as you give appropriate credit to the original author(s) and the source, provide a link to the Creative Commons licence, and indicate if changes were made. The images or other third party material in this article are included in the article's Creative Commons licence, unless indicated otherwise in a credit line to the material. If material is not included in the article's Creative Commons licence and your intended use is not permitted by statutory regulation or exceeds the permitted use, you will need to obtain permission directly from the copyright holder. To view a copy of this licence, visit http://creativecommons.org/licenses/ by/4.0\%.

Publisher's Note Springer Nature remains neutral with regard to jurisdictional claims in published maps and institutional affiliations.

\section{REFERENCES}

[1] Timothy Candy and Sebastian Herr, Transference of bilinear restriction estimates to quadratic variation norms and the Dirac-Klein-Gordon system, Anal. PDE 11 (2018), no. 5, 1171-1240.

[2] Yonggeun Cho and Sanghyuk Lee, Strichartz estimates in spherical coordinates, Indiana Univ. Math. J. 62 (2013), no. 3, 991-1020.

[3] A. V. Faminskiŭ, The Cauchy problem for the Zakharov-Kuznetsov equation, Differentsial' nye Uravneniya 31 (1995), no. 6, 1070-1081, 1103.

[4] Axel Grünrock and Sebastian Herr, The Fourier restriction norm method for the Zakharov-Kuznetsov equation, Discrete Contin. Dyn. Syst. 34 (2014), no. 5, 2061-2068.

[5] Sebastian Herr and Shinya Kinoshita, Subcritical well-posedness results for the Zakharov-Kuznetsov equation in dimension three and higher, arXiv:2001.09047.

[6] Markus Keel and Terence Tao, Endpoint Strichartz estimates, Amer. J. Math. 120 (1998), no. 5, 955-980.

[7] S. Kinoshita, Global Well-posedness for the Cauchy problem of the Zakharov-Kuznetsov equation in 2D, arxiv: 1905.01490, accepted for publication in Annales de l'Institut Henri Poincaré - Analyse Non Linéaire.

[8] Herbert Koch and Daniel Tataru, Dispersive estimates for principally normal pseudodifferential operators, Comm. Pure Appl. Math. 58 (2005), no. 2, 217-284.

[9] Felipe Linares and Ademir Pastor, Well-posedness for the two-dimensional modified ZakharovKuznetsov equation, SIAM J. Math. Anal. 41 (2009), no. 4, 1323-1339. 
[10] Felipe Linares and Jean-Claude Saut, The Cauchy problem for the 3D Zakharov-Kuznetsov equation, Discrete Contin. Dyn. Syst. 24 (2009), no. 2, 547-565.

[11] Luc Molinet and Didier Pilod, Bilinear Strichartz estimates for the Zakharov-Kuznetsov equation and applications, Ann. Inst. H. Poincaré Anal. Non Linéaire 32 (2015), no. 2, 347-371.

[12] Francis Ribaud and Stéphane Vento, Well-posedness results for the three-dimensional ZakharovKuznetsov equation, SIAM J. Math. Anal. 44 (2012), no. 4, 2289-2304.

[13] V. E. Zakharov and E. A. Kuznetsov, Three-dimensional solitons, Sov. Phys. JETP 39 (1974), no. 2, 285-286.

\author{
Sebastian Herr and Shinya Kinoshita \\ Fakultät für Mathematik \\ Universität Bielefeld \\ Postfach 100131 \\ 33501 Bielefeld \\ Germany \\ E-mail: herr@math.uni-bielefeld.de \\ Shinya Kinoshita \\ E-mail: kinoshita@math.uni-bielefeld.de
}

